\title{
Majorization by starlike functions
}

\author{
Öznur Özkan Kılıça,*, Osman Altıntaş ${ }^{\mathrm{b}}$ \\ ${ }^{a}$ Başkent University, Department of Statistics and Computer Sciences, Bağlıca, 06810, Ankara, Turkey. \\ ${ }^{b}$ Başkent University, Department of Mathematics Education, Bağlıca, 06810, Ankara, Turkey.
}

Communicated by E. Savaş

\begin{abstract}
The main object of this paper is to investigate some majorization problems involving the subclass $S(\alpha, A, B)$ of starlike functions in the open unit disk U. Relevant connections of the results presented here with those given by earlier workers on the subject are also indicated. (C)2017 All rights reserved.
\end{abstract}

Keywords: Analytic function, starlike function, convex function, subordination, majorization, Quasi-subordination. 2010 MSC: 30C45.

\section{Introduction}

Let $A$ denote the class of functions of the form

$$
f(z)=z+\sum_{n=2}^{\infty} a_{n} z^{n},
$$

which are analytic in the open unit disk

$$
\mathrm{U}=\{z: z \in \mathbb{C} \text { and }|z|<1\} .
$$

Definition 1.1. For two functions $f$ and $g$, which are analytic in $U$, the function $f$ is said to be subordinate to $\mathrm{g}$, written as

$$
f \prec g \quad \text { or } \quad f(z) \prec g(z)
$$

if there exists a Schwarz function $w$ analytic in $\mathrm{U}$, with

$$
\omega(0)=0 \text { and }|\omega(z)|<1 \quad(z \in \mathrm{U})
$$

and such that

$$
f(z)=g(\omega(z)) \quad(z \in U) .
$$

In particular, if the function $\mathrm{g}$ is univalent in $\mathrm{U}$, the above subordination is equivalent to

$$
f(0)=g(0) \quad \text { and } \quad f(U) \subset g(U) .
$$

\footnotetext{
*Corresponding author

Email addresses: oznur@baskent.edu.tr (Öznur Özkan Kılıç), oaltintas@baskent.edu .tr (Osman Altıntaş) doi:10.22436/jnsa.010.03.29
} 
Definition 1.2. For two functions $f$ and $g$, which are analytic in $U$, the function $f$ is said to be majorized to $\mathrm{g}$, written as

$$
f<<\text { or } f(z)<<g(z)
$$

if there exists a function $\varphi$ analytic in $\mathrm{U}$, with

$$
|\varphi(z)|<1 \quad(z \in \mathrm{U})
$$

and such that

$$
f(z)=\varphi(z) g(z) \quad(z \in \mathrm{U})
$$

(see MacGregor [6]).

The majorization is closely related to the concept of quasi-subordination between analytic functions, which was considered recently by (for example) Altıntaş and Owa [3]. Some majorization problems were studied by Altıntaş et al. in [4,5]. Therefore, various subclasses of univalent functions in U were studied by Akgul in [1, 2].

We purpose to investigate the majorization problems associated with the class $S(\alpha, A, B)$ of starlike functions.

Definition 1.3. We denote by $S(\alpha, A, B)$ the class of functions satisfying the condition

$$
\frac{z f^{\prime}(z)}{f(z)}+\alpha z\left(\frac{z f^{\prime}(z)}{f(z)}\right)^{\prime} \prec \frac{1+A z}{1+B z}
$$

$(z \in \mathrm{U}, \mathrm{f} \in \mathrm{A}, 0 \leqslant \alpha \leqslant 1,-1 \leqslant B<A \leqslant 1)$.

Clearly, we have the following relationships:

- $S(0,1,-1)=S^{*}$ is the class of starlike functions;

- $\mathrm{S}(0,0,-1)=\mathrm{C}$ is the class of convex functions;

- $S(0,1-2 \alpha,-1)=S^{*}(\alpha)$ is the class of starlike functions of order $\alpha,(0 \leqslant \alpha<1)$;

- $S(0,1-\alpha,-1)=C(\alpha)$ is the class of convex functions of order $\alpha,(0 \leqslant \alpha<1)$.

\section{Majorization problems for the class $S(\alpha, A, B)$}

We first state and prove the following Lemma 2.1.

Lemma 2.1 ([9]). If the function $h(z)=1+\sum_{n=1}^{\infty} c_{n} z^{n}$ is analytic in $\mathrm{U}$ and satisfies the condition

$$
h(z) \prec \frac{1+A z}{1+B z} \quad(z \in U,-1 \leqslant B<A \leqslant 1),
$$

then

$$
\operatorname{Reh}(z)>\frac{1-A}{1-B}=\beta .
$$

Proof. Using (1.1) and (2.1) we have

$$
h(z)=\frac{1+A \omega(z)}{1+B \omega(z)} \quad(\omega(0)=0,|\omega(z)|<1)
$$

and

$$
|\omega(z)|=\left|\frac{h(z)-1}{A-B h(z)}\right|,
$$

for $h(z)=u+i v$. 
Since $|h(z)|^{2} \geqslant[\operatorname{Re} h(z)]^{2}$, we have

$$
\left(1-B^{2}\right) u^{2}-2(1-A B) u+1-A^{2}<0,
$$

which implies that

$$
\frac{1-A}{1-B}<u=\operatorname{Reh}(z)<\frac{1+A}{1+B}
$$

Lemma 2.2 ([8]). If the function $p(z)=1+\sum_{n=1}^{\infty} p_{n} z^{n}$ is analytic in $\mathrm{U}$ and satisfies the condition

$$
\operatorname{Re}\left(\mathrm{p}(z)+\alpha z \mathrm{p}^{\prime}(z)\right)>\beta,
$$

then

$$
\operatorname{Re} p(z)>\frac{\alpha+2 \beta}{\alpha+2} \quad(0 \leqslant \alpha \leqslant 1,0 \leqslant \beta<1) .
$$

Theorem 2.3. Let the function $\mathrm{f}(z)$ be in the class $A$ and suppose that $\mathrm{g} \in \mathrm{S}(\alpha, \mathrm{A}, \mathrm{B})$. If $\mathrm{f}(z)$ is majorized by $\mathrm{g}(z)$ in $\mathrm{U}$, then

$$
\left|f^{\prime}(z)\right| \leqslant\left|g^{\prime}(z)\right| \quad\left(|z| \leqslant r_{1}\right)
$$

where

$$
r_{1}=r_{1}(\alpha, A, B)=\frac{3+|1-2 \gamma|-\sqrt{|1-2 \gamma|^{2}+2|1-2 \gamma|+9}}{2|1-2 \gamma|}
$$

and

$$
\gamma=\frac{\alpha(1-B)+2(1-A)}{(\alpha+2)(1-B)} \quad(0 \leqslant \alpha \leqslant 1,-1 \leqslant B<A<1) .
$$

Proof. Since $g \in S(\alpha, A, B)$, if we let

$$
\frac{z g^{\prime}(z)}{g(z)}=p(z) \text { and }\left(p(z)+\alpha z p^{\prime}(z)\right)=h(z)
$$

and $\beta=\frac{1-A}{1-B}$, then using (1.2), (2.2), (2.3), and (2.4) we find

$$
\operatorname{Re} \frac{z g^{\prime}(z)}{g(z)}>\frac{\alpha+2 \beta}{\alpha+2}
$$

Letting $\gamma=\frac{\alpha+2 \beta}{\alpha+2}$, we obtain

$$
\frac{z g^{\prime}(z)}{g(z)}=\frac{1-(1-2 \gamma) \omega(z)}{1+\omega(z)}
$$

where $\omega(0)=0$ and $|\omega(z)|<1$.

Hence we find the inequality

$$
|g(z)| \leqslant\left(\frac{(1+|z|)|z|}{1-|1-2 \gamma||z|}\right)\left|g^{\prime}(z)\right| \quad(z \in \mathrm{U}) .
$$

Since $f(z)$ is majorized by $g(z)$ in $U$, from (1.1) we have

$$
f^{\prime}(z)=\varphi(z) g^{\prime}(z)+\varphi^{\prime}(z) g(z) .
$$

We know that $\varphi(z)$ satisfies the inequality (Nehari, [7, p.168])

$$
\left|\varphi^{\prime}(z)\right| \leqslant \frac{1-|\varphi(z)|^{2}}{1-|z|^{2}} \quad(z \in \mathrm{U})
$$


and using (2.7) and (2.9) in (2.8), we get

$$
\left|f^{\prime}(z)\right| \leqslant\left(|\varphi(z)|+\frac{1-|\varphi(z)|^{2}}{1-|z|^{2}} \frac{(1+|z|)|z|}{1-|1-2 \gamma||z|}\right)\left|g^{\prime}(z)\right|,
$$

which upon setting

$$
|z|=r \quad \text { and } \quad|\varphi(z)|=\mu \quad(0 \leqslant \mu \leqslant 1)
$$

we have the inequality

$$
\left|f^{\prime}(z)\right| \leqslant \frac{\Theta(\mu)}{(1-r)(1-|1-2 \gamma| r)}\left|g^{\prime}(z)\right| \quad(z \in U),
$$

where the function $\Theta(\mu)$ defined by

$$
\Theta(\mu)=-r \mu^{2}+(1-r)(1-|1-2 \gamma| r) \mu+r \quad(0 \leqslant \mu \leqslant 1)
$$

takes the maximum value at $\mu=1$ with $r=r_{1}(\gamma)$ given by (2.5).

Furthermore, if $0 \leqslant q \leqslant r_{1}(\gamma)$ is given by (2.5), then we have

$$
\Lambda(\mu) \leqslant \Lambda(1)=(1-r)(1-|1-2 \gamma| r) \quad\left(0 \leqslant \mu \leqslant 1,0 \leqslant q \leqslant r_{1}(\gamma)\right) .
$$

Hence, upon setting $\mu=1$ in (2.10), we conclude that the inequality in (2.5) holds true for $|z| \leqslant r_{1}(\gamma)$ and is given by (2.6). The proof of Theorem 2.4 is based on Lemma 1 in [4],

$$
\mathrm{f} \in \mathrm{C}(\gamma) \Longrightarrow \mathrm{f} \in \mathrm{S}\left(\frac{1}{2} \gamma\right)
$$

Theorem 2.4. Let the function $\mathrm{f}(z)$ be analytic in $\mathrm{U}$ and suppose that $\mathrm{g} \in \mathrm{C}(\gamma)$. If $\mathrm{f}(z)$ is majorized by $\mathrm{g}(z)$ in $\mathrm{U}$, then

$$
\left|f^{\prime}(z)\right| \leqslant\left|g^{\prime}(z)\right| \quad\left(|z| \leqslant r_{2}\right),
$$

where

and

$$
r_{2}=r_{2}(\alpha, A, B)=\frac{3+|1-\gamma|-\sqrt{|1-\gamma|^{2}+2|1-\gamma|+9}}{2|1-\gamma|}
$$

$$
\gamma=\frac{\alpha(1-B)+2(1-A)}{(\alpha+2)(1-B)} \quad(0 \leqslant \alpha \leqslant 1,-1 \leqslant B<A \leqslant 1) .
$$

Proof. Upon replacing $\gamma$ in Theorem 2.3 by $\frac{1}{2} \gamma$, the conclusion follows.

Letting special values for $\alpha, A, B$ we have the following corollaries.

Corollary 2.5. If $\mathrm{g} \in \mathrm{S}(\alpha, 1,-1)$ and $\mathrm{f}(\mathrm{z})$ is majorized by $\mathrm{g}(\mathrm{z})$ in $\mathrm{U}$, then

$$
\left|f^{\prime}(z)\right| \leqslant\left|g^{\prime}(z)\right| \quad(|z| \leqslant r),
$$

where

$$
|z| \leqslant r=\frac{8+2 \alpha-\sqrt{8 \alpha^{2}+32 \alpha+48}}{2(2-\alpha)} \quad(0 \leqslant \alpha \leqslant 1) .
$$

Proof. We let $A=1, B=-1$ in (2.6) and $\gamma=\frac{\alpha}{\alpha+2}$ in Theorem 2.3.

Corollary 2.6. If $\mathrm{g} \in \mathrm{S}(0,1,-1)$ and $\mathrm{f}(\mathrm{z})$ is majorized by $\mathrm{g}(\mathrm{z})$ in $\mathrm{U}$, then

$$
\left|f^{\prime}(z)\right| \leqslant\left|g^{\prime}(z)\right| \quad(|z| \leqslant r),
$$

where

$$
|z| \leqslant r=2-\sqrt{3}
$$


Proof. We let $\alpha=0, A=1, B=-1$ in (2.6) and $\gamma=0$ in Theorem 2.3.

Corollary 2.7. If $\mathrm{g} \in \mathrm{S}(\alpha, 0,-1)$ and $\mathrm{f}(z)$ is majorized by $\mathrm{g}(z)$ in $\mathrm{U}$, then

$$
\left|f^{\prime}(z)\right| \leqslant\left|g^{\prime}(z)\right| \quad(|z| \leqslant r),
$$

where

$$
|z| \leqslant r=\frac{2 \alpha+3-\sqrt{3 \alpha^{2}+10 \alpha+9}}{\alpha} \quad(0 \leqslant \alpha \leqslant 1) .
$$

Proof. We let $A=0, B=-1$ in (2.6) and $\gamma=\frac{\alpha+1}{\alpha+2}$ in Theorem 2.3.

Corollary 2.8. If $\mathrm{g} \in \mathrm{S}(1,1,-1)$ and $\mathrm{f}(\mathrm{z})$ is majorized by $\mathrm{g}(\mathrm{z})$ in $\mathrm{U}$, then

$$
\left|f^{\prime}(z)\right| \leqslant\left|g^{\prime}(z)\right| \quad(|z| \leqslant r),
$$

where

$$
|z| \leqslant r=5-\sqrt{22}
$$

Proof. We let $\alpha=1, \mathrm{~A}=1, \mathrm{~B}=-1$ in (2.6) and $\gamma=\frac{1}{3}$ in Theorem 2.3.

Corollary 2.9. If $\mathrm{g} \in \mathrm{C}(\alpha, 1,-1)$ and $\mathrm{f}(\mathrm{z})$ is majorized by $\mathrm{g}(\mathrm{z})$ in $\mathrm{U}$, then

$$
\left|f^{\prime}(z)\right| \leqslant\left|g^{\prime}(z)\right| \quad(|z| \leqslant r),
$$

where

$$
|z| \leqslant r=\frac{8+3 \alpha-\sqrt{9 \alpha^{2}+40 \alpha+48}}{4} \quad(0 \leqslant \alpha \leqslant 1) .
$$

Proof. We let $A=1, B=-1$ in (2.6) and $\gamma=\frac{\alpha}{\alpha+2}$ in Theorem 2.4.

Corollary 2.10. If $\mathrm{g} \in \mathrm{C}(0,1,-1)$ and $\mathrm{f}(z)$ is majorized by $\mathrm{g}(z)$ in $\mathrm{U}$, then

$$
\left|f^{\prime}(z)\right| \leqslant\left|g^{\prime}(z)\right| \quad(|z| \leqslant r),
$$

where

$$
|z| \leqslant r=2-\sqrt{3} .
$$

Proof. We let $\alpha=0, A=1, B=-1$ in (2.6) and $\gamma=0$ in Theorem 2.4.

Corollary 2.11. If $\mathrm{g} \in \mathrm{C}(\alpha, 0,-1)$ and $\mathrm{f}(z)$ is majorized by $\mathrm{g}(z)$ in $\mathrm{U}$, then

$$
\left|f^{\prime}(z)\right| \leqslant\left|g^{\prime}(z)\right| \quad(|z| \leqslant r),
$$

where

$$
|z| \leqslant r=\frac{7+3 \alpha-\sqrt{9 \alpha^{2}+38 \alpha+41}}{2} \quad(0 \leqslant \alpha \leqslant 1) .
$$

Proof. We let $A=0, B=-1$ in (2.6) and $\gamma=\frac{\alpha+1}{\alpha+2}$ in Theorem 2.4.

Corollary 2.12. If $\mathrm{g} \in \mathrm{C}(1,1,-1)$ and $\mathrm{f}(z)$ is majorized by $\mathrm{g}(z)$ in $\mathrm{U}$, then

$$
\left|f^{\prime}(z)\right| \leqslant\left|g^{\prime}(z)\right| \quad(|z| \leqslant r),
$$

where

$$
|z| \leqslant r=\frac{11-\sqrt{97}}{4} .
$$

Proof. We let $\alpha=1, \mathrm{~A}=1, \mathrm{~B}=-1$ in (2.6) and $\gamma=\frac{1}{3}$ in Theorem 2.4. 
Corollary 2.13. If $\mathrm{g} \in \mathrm{S}(0,0,-1)$ and $\mathrm{f}(z)$ is majorized by $\mathrm{g}(z)$ in $\mathrm{U}$, then

$$
\left|f^{\prime}(z)\right| \leqslant\left|g^{\prime}(z)\right| \quad(|z| \leqslant r),
$$

where

$$
|z| \leqslant r=\frac{1}{3} .
$$

Remark 2.14. $\mathrm{S}(0,0,-1)=\mathrm{S}^{*}\left(\frac{1}{2}\right)$ and $\mathrm{C} \subset \mathrm{S}^{*}\left(\frac{1}{2}\right)$.

\section{Acknowledgment}

The authors would like to express their thanks to the referee and the editors for their helpful comments and advices.

\section{References}

[1] A. Akgül, A new subclass of meromorphic functions defined by Hilbert space operator, Honam Math. J., 38 (2016), 495-506. 1

[2] A. Akgül, A new subclass of meromorphic functions with positive and fixed second coefficients defined by the rafid-operator, Commun. Fac. Sci. Univ. Ank. Series A1, 66 (2017), 1-13. 1

[3] O. Altıntaş, S. Owa, Majorizations and quasi-subordinations for certain analytic functions, Proc. Japan Acad. Ser. A Math. Sci., 68 (1992), 181-185. 1

[4] O. Altıntaş, Ö. Özkan, H. M. Srivastava, Majorization by starlike functions of complex order, Complex Variables Theory Appl., 46 (2001), 207-218. 1, 2

[5] O. Altıntaş, H. M. Srivastava, Some majorization problems associated with p-valently starlike and convex functions of complex order, East Asian Math. J., 17 (2001), 175-183. 1

[6] T. H. MacGregor, Majorization by univalent functions, Duke Math. J., 34 (1967), 95-102. 1.2

[7] Z. Nehari, Conformal mapping, McGraw-Hill Book Co., Inc., New York, Toronto, London, (1952). 2

[8] S. Owa, C. Y. Shen, Certain subclass of analytic functions, Math. Japan., 34 (1989), 409-412. 2.2

[9] H. M. Srivastava, O. Altıntaş, S. K. Serenbay, Coefficient bounds for certain subclasses of starlike functions of complex order, Appl. Math. Lett., 24 (2011), 1359-1363. 2.1 